\title{
New Laser Techniques for Time-Resolved Spectroscopy
}

\author{
A. H. Zewail \\ Arthur Amos Noyes Laboratory of Chemical Physics* \\ California Institute of Technology \\ Pasadena, California 91125
}

\begin{abstract}
A novel spectroscopic tool is presented for measuring the incoherent resonance decay (IRD) of selectively $(6 \mathrm{MHz})$ prepared electronic states. The method utilizes electro-optic switching of a single laser mode that is on or off resonance with respect to the homogeneous molecular packets in the excited ensemble. The technique is applied to a variety of systems: gases at low and high pressures, molecular beams, and solids at low temperatures. The coherent transient of solids observed in the forward direction of the laser gives the phase memory (optical $T_{2}$ processes) while the IRD measures directly the radiative and radiationless decay (optical $T_{1}$ processes), and the optical transition moment between the ground state and the prepared electronic state. The theory of coherent and incoherent states is also given, and related to the different aspects of time-resolved spectroscopy of molecular beams and solids.
\end{abstract}

\section{Introduction}

The phenomenological understanding for the decay of excited levels following incoherent optical excitation has been well known since 1930 when Weisskopf and Wigner ${ }^{1}$ showed that the addition of a damping matrix to the Schrödinger equation gives a Lorentzian ${ }^{2}$ resonance centered around the transition frequency $\omega_{0}=E_{0} / \hbar$. One might therefore conclude that careful measurements of the linewidth of optical transitions will give the dynamics ${ }^{3}$ of excited levels. Unfortunately, these levels are not isolated from the rest of the ensemble which may or may not be homogeneous.

In gases, the molecular packets (i.e., the distribution of molecular states that are homogeneous) are nonstationary (i.e., translating their energy); thus the Doppler resonance is a weighted statistical distribution for the population amongst all packets. Therefore, to characterize the dynamics one must know the actual width of the homogeneous packets as well as the relative importance of pressure-induced broadening and pressure-induced velocity changes during the optical pumping.

In solids, although the sites are not moving, the problem is similar because Doppler solids do exist. In other words, different molecules (or ions) experience different crystal fields and thus are located at slightly shifted energy which appear in the frame of $\omega_{0}$ as being moving. ${ }^{4}$ Equivalently one says that the crystal ensemble is inhomogeneous.

With the advances in laser technology, spectroscopists have tried to narrow the frequency width of these inhomogeneous optical resonances, using different methods. All these methods

Most of this paper is based on a lecture presented at the 20th Annual Symposium of SPIE, August 1976.

*Contribution No. 5459.

$\overline{\text { Paper } 1412}$ received October 28, 1976, revised December 1, 1976. extract a linewidth that is smaller than the inhomogeneous width. However, the contribution of the different coherent and incoherent decay processes cannot be extracted in a direct way. To understand fully the many interactions that lead to spectral broadening of the primary states, one is therefore forced to answer the following questions:

(1) What are the radiative and nonradiative decays (optical $\mathrm{T}_{1}$ processes) that contribute to the width of the resonance?

(2) Are excited electronic states optically coherent? In other words, what is the phase memory (optical $T_{2}$ processes of excited states in gases and solids?

(3) How do optical $T_{1}$ and $T_{2}$ processes ${ }^{5}$ depend on the vibronic energy?

This paper will first outline succinctly the theoretical recipes needed for describing the coherent and incoherent properties of excited states. Second, it will be shown that the technique of incoherent resonance decay (IRD) is very sensitive to the relaxation processes of the excited ensemble. It also replaces other techniques that are limited by the cross section, and the quality of the material. Finally, we will discuss our observation of the coherent ringing and decay in solids and beams which manifest the finite phase memory and the radiationless relaxations that follow the laser excitation.

\section{What is Coherence?}

Coherence is a manifestation of the phenomenon of interference in quantum mechanics. In other words, if we have two electronic states (say, the ground state $\psi_{\mathrm{g}}$ and an excited electronic state $\psi_{\mathrm{e}}$ ) and we manage to form a superposition of these two states, a coherent state will be formed:

$$
\psi(r, t)=C_{g} e^{-i \omega_{g} t} \psi_{g}(r)+C_{e} e^{-i \omega_{e} t} \psi_{e}(r)
$$

where $\mathrm{C}_{\mathrm{g}} \mathrm{C}_{\mathrm{g}}$ * is the probability of finding the system in the $\mathrm{g}$ state and $\omega_{\mathrm{g}}=\mathrm{E}_{\mathrm{g}} / \mathrm{h}$. This coherent state has two interesting properties.

(a) The density matrix for an ensemble of such levels is nondiagonal, i.e., a pure state is created with

$$
\stackrel{\rho}{\approx}=\left(\begin{array}{ll}
\mathrm{C}_{\mathrm{g}} \mathrm{C}_{\mathrm{g}}{ }^{*} & \mathrm{C}_{\mathrm{g}} \mathrm{C}_{\mathrm{e}}{ }^{*} \mathrm{e}^{+\mathrm{i} \omega_{0} \mathrm{t}} \\
\text { C.C. } & \mathrm{C}_{\mathrm{e}} \mathrm{C}_{\mathrm{e}}{ }^{*}
\end{array}\right)=\left(\begin{array}{ll}
\rho_{\mathrm{gg}} & \rho_{\mathrm{ge}} \\
\rho_{\mathrm{eg}} & \rho_{\mathrm{ee}}
\end{array}\right)
$$

where $\omega_{0}$ is the resonance frequency and C.C. denotes the complex conjugate. 
(b) The expectation value for the moment $\mu$ is oscillating in time. Assuming that the ground and excited states have no permanent dipole moment,

$$
\langle u\rangle=C_{g} C_{e}^{*} \mu_{g e} e^{i \omega_{0} t}+\text { C.C. }
$$

This quantum mechanical dipole moment radiates light in the same way as a classical oscillating "charge on a spring." Indeed, this simple description explains the many interesting and exciting experiments (such as photon echoes) done recently in atomic, ionic, and molecular systems.

We notice that if the excited ensemble can be described by the above pure density matrix, the system is coherent for the entire lifetime of the state. On the other hand, if the ensemble is impure, a phase shift will exist among the sub-ensembles (i.e., the different two-levels) such that coherence will be lost. Naturally the intermediate limit is challenging for reasons which raise the following two questions: (i) how can one measure the degree of coherence in mixed states, and (ii) what causes the creation of the impure (partially coherent or completely incoherent) state?

\section{Two-Level System in a Laser Field}

The treatment of the interaction between a pulse of radiation (width $t_{p}<T_{1}, T_{2}$ ) and a two-level system has been well known since 1946 when Bloch $^{6}$ predicted the existence of phase coherence in a spin ensemble. Hahn ${ }^{7}$ in 1950 demonstrated in a classical experiment the dephasing and refocusing of the ensemble moments as a result of finite coherence time that is longer than Rabi's oscillation time in the rotating frame. Hahn's discovery showed clearly that the reversibility of free induction decay exists. The extension of such ideas to the optical region was shown by Kurnit et al. ${ }^{8}$ In their experiments an echo resulted from the in-plane refocusing of the homogeneous optical moments. This discovery has triggered similar experiments $^{9}$ done on other systems. The optical nutation which is another coherent optical effect was observed first by Hocker and Tang ${ }^{10}$ in $\mathrm{SF}_{6}$. Brewer and his co-workers ${ }^{11}$ have introduced the elegant technique of molecular and laser switching to observe the different coherent transients of gases. The method which monitors the signal in the forward direction of the laser is different from the IRD technique.* At low temperatures, both the IRD and the coherent transient of solids were observed* by us.

In these nonlinear spectroscopic techniques, the diagonal and off-diagonal elements of the density matrix $(\underset{\widetilde{c}}{\rho})$ are being monitored in optically thin samples. Being optically thin ensures that there are no propagation effects, such as self-induced transparency. ${ }^{12}$ The sample emitting cooperatively resembles Dicke's superradiance or, more specifically, the linear combination (molecules plus field) of Dicke's states. ${ }^{13}$ Therefore, the coupling between Maxwell's equations of the radiation field and the Schroddinger representation ${ }^{14}$ of the two-level system becomes straightforward.

Defining the total decay time constant for the two levels as $\mathrm{T}_{1 \mathrm{e}}$ and $\mathrm{T}_{1} \mathrm{~g}$, one can show that the rate of change of the elements of the density matrix is given by

$$
-\dot{\rho}_{\mathrm{ee}}=\frac{\rho_{\mathrm{ee}}}{\mathrm{T}_{1 \mathrm{e}}}-\hat{\rho}
$$

\footnotetext{
*A. H. Zewail, T. E. Orlowski and D. R. Dawson, Chem. Phys. Lett., 44, 379 (1976); A. H. Zewail, SPIE Proceedings 82:43 (1976).
}

$$
\begin{aligned}
&-\dot{\rho}_{\mathrm{gg}}= \frac{\rho_{\mathrm{gg}}}{\mathrm{T}_{1 \mathrm{~g}}}+\hat{\rho} \\
&-\dot{\rho}_{\mathrm{eg}}=\left\{\mathrm{i}\left[\omega_{0}+\delta(\mathrm{t})\right]+\left[1 / 2\left(\mathrm{~T}_{1 \mathrm{e}}^{-1}+\mathrm{T}_{1 \mathrm{~g}}^{-1}\right)\right]\right\} \rho_{\mathrm{eg}} \\
&-\mathrm{i} / \hbar \mathrm{V}_{\mathrm{eg}}\left(\rho_{\mathrm{ee}}-\rho_{\mathrm{gg}}\right) \\
&-\hat{\rho}_{=} \mathrm{i} / \hbar \mathrm{V}_{\mathrm{eg}} \rho_{\mathrm{ge}}+\text { c.c. }
\end{aligned}
$$

The above equations imply first that there exist differences in the decay if one monitors the diagonal and/or off-diagonal elements, i.e., the polarization component or the population difference between the two levels $\left(\rho_{\mathrm{ee}}-\rho_{\mathrm{gg}}\right)$. The latter is, of course, proportional to $R_{3}$ in the FVH picture. ${ }^{14}$ Second, the dipole dephasing is incorporated in Eq. (6) as a fluctuating term $\delta$ that gives rise to a decay constant $1 / \mathrm{T}_{2}$ which can be added to the $1 / T_{1}$ term. This is true if one assumes a simple correlation function for the dephasing process in the gaseous or the solid ensemble when the interaction $\mathrm{V}\left(\mathrm{V}=-\mu \cdot \mathrm{E}_{0} \cos \omega \mathrm{t}\right)$ couples the radiation field (of frequency $\omega$ ) with the system of moment $\mu$. In our case, the laser field in the optical region couples with the electric dipoles that are generated from the transition between the ground state and a narrow optical electronic state. It is this coupling that creates the in-phase and the quadrature components of the polarization which evolve in time as follows:

$$
\begin{aligned}
& \dot{\mathrm{R}}_{1}=-\Delta \mathrm{R}_{2}-\gamma \mathrm{R}_{1} \\
& \dot{\mathrm{R}}_{2}=\Delta \mathrm{R}_{1}-\gamma \mathrm{R}_{2}+\left(\frac{\mu \mathrm{E}_{0}}{\hbar}\right) \mathrm{R}_{3} \\
& \dot{\mathrm{R}}_{3}=\left(-\gamma_{\mathrm{e}} \rho_{\mathrm{ee}}+\gamma_{\mathrm{g}} \rho_{\mathrm{gg}}\right)-\left(\frac{\mu \mathrm{E}_{0}}{\hbar}\right) \mathrm{R}_{2} .
\end{aligned}
$$

$\gamma_{\mathrm{e}}$ and $\gamma_{\mathrm{g}}$ are the decay rate constants of levels e and $\mathrm{g}$, respectively, while $\gamma=\left(\gamma_{\mathrm{e}}+\gamma_{\mathrm{g}}\right) / 2+1 / \mathrm{T}_{2} . \Delta$ is the amount off-resonance the single mode of the laser is with respect to the transition frequency of the primary state in the optical rotating frame. $R_{1}$ and $R_{2}$ are directly related to $\rho_{\mathrm{eg}}$ and $\rho_{\mathrm{ge}}$. The above Bloch-type equations imply that a macroscopic polarization $\mathrm{P}$ reacts back on the applied field and causes a dispersion or absorption (or gain) depending on the relative phase between $\mathrm{P}$ and the applied field. Moreover, the equations are reminiscent of the torque equation for the spin $1 / 2$ problem since

$$
\frac{d \vec{P}}{d t}=K\left(\vec{P} \times E_{e f f}\right)
$$

$\mathrm{K}$ is related to the dipole matrix element and $\overrightarrow{\mathrm{E}}_{\text {eff }}$ is the effective field. In the rotating frame and on-resonance, the population vector will be driven (nutation) in a plane perpendicular to the $\mu \mathrm{E}_{0}$ axis, depending on the strength of the moment and the laser field. Of course, when $E_{0}$ is switched off, $R_{3}$ will decay by its usual decay time constant. Hence, both $T_{1}$ and $T_{2}$ optical processes can be obtained in a solid or a gas when certain sub-ensembles are switched on and off resonance.

\section{The Incoherent Resonance Decay (IRD) In Gases and Solids}

In the IRD experiments, an intense single mode tunable dye laser was used for the excitation. An $\mathrm{Ar}^{+}$ion laser (Spectra Physics Model 170; total power $=18$ watts) was used to pump a free jet stream dye laser. The dye solution was rhodamine 6G in ethylene glycol. A modified Spectra Physics (Model 580 A) 
dye laser was used so a single mode could be switched out of the transition resonance frequency by an electro-optic element. Special attention was given to the alignment procedure so the dispersion of the crystal in the electric field was caused by the modulation of the refractive index along the principal axes of the dielectric ellipsoid. The modulation is done transversely and is different from the switching procedure of Telle and Tang ${ }^{15}$ used for very rapid tuning of cw dye lasers. The switching is clearly demonstrated in our experiments as shown in Figure 1. From the dispersion frequency we obtained an electro-optic dispersion for the $\mathrm{AD} * \mathrm{P}$ crystal of $1.4 \mathrm{MHz} / \mathrm{V}$. The net laser power of the single mode can be varied up to $90 \mathrm{~mW}$ and the field is linearly polarized.

The single mode beam was split so both the frequency of the transition and the quality of the single mode can be checked during the experiments (see Figure 1). The single-mode spectrum was monitored with a Fabry-Perot interferometer that is scanned to allow the observation of on-resonance and off- resonance mode structure of the laser beam. Most of the laser beam intensity was used in the forward direction for the excitation of the sample. For the study of low pressure gases $\left(\mathrm{I}_{2}\right.$ and $\mathrm{NO}_{2}$ ), a sample tube with optical windows was used. A cold finger at the side of the tube was used to control the pressure of the gas. For solid state experiments, the crystal was oriented in a liquid helium Dewar that can be pumped to temperatures below the $\lambda$-point of liquid helium.

The emission was collimated at a right angle to the exciting beam with lenses of proper $f$ numbers, and focused on a cooled photomultiplier (EMI-9558). The photomultiplier was magnetically and electrostatically shielded. Since noise will be introduced because of the laser light scattering, a sharp cutoff filter was placed in front of the phototube. The output of the photomultiplier was terminated in a boxcar integrator (PAR Model 162) whose gate width was adjusted between 5-50 nsec. The photomultiplier was coupled $\mathrm{AC}$ into the boxcar and the output was traced on an X-Y recorder. Typical results are shown

\section{IRD and COR Experimental Setup}
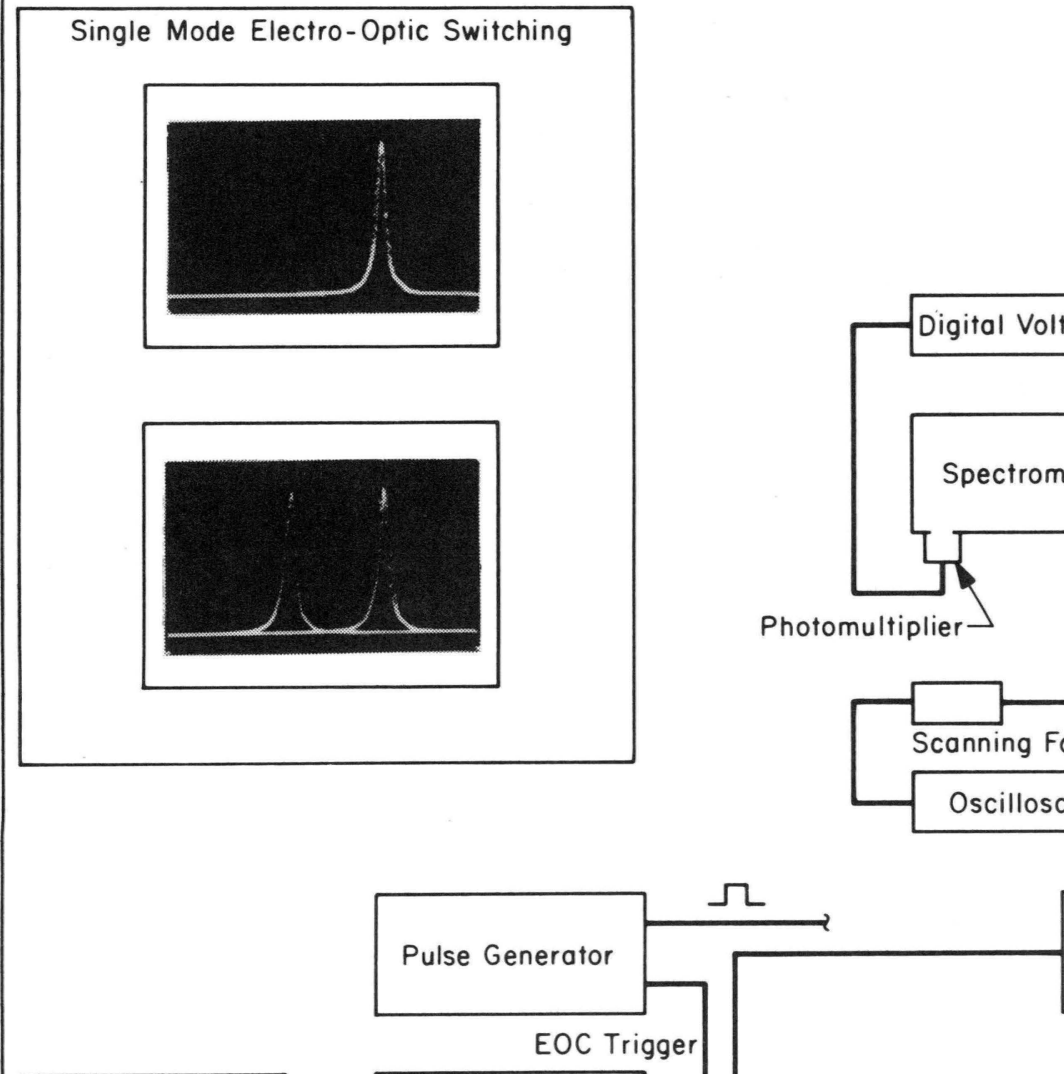

\section{Cooled \\ Photomultiplier}
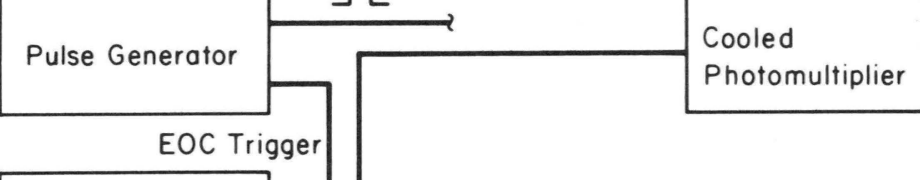

X-Y Recorder
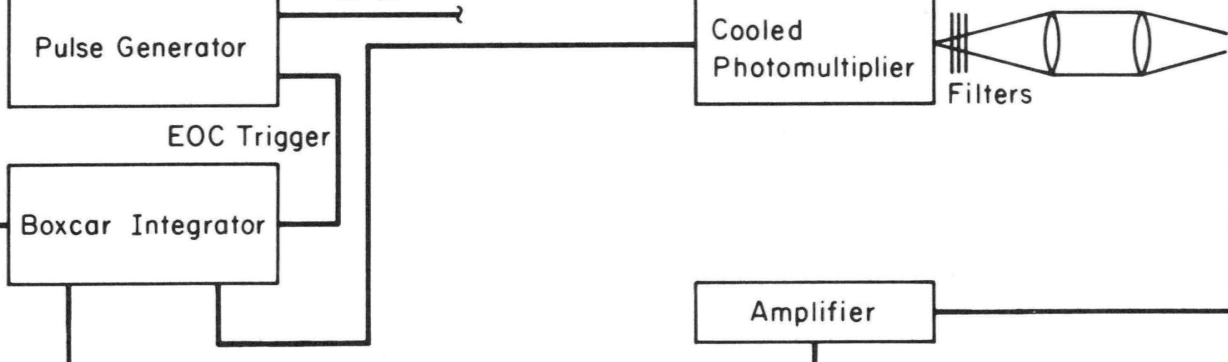

Sample

AC Bandpass Filter

Figure 1. A schematic diagram of the experimental setup for the incoherent resonance decay (IRD) and coherent optical resonance (COR) experiments. The insert in the figure shows the single mode of the $\mathrm{cw}$ dye laser when the voltage on the electro-optic crystal (1.4 $\mathrm{MHz} / \mathrm{V})$ is off and on. The mode spectrum was monitored using an interferometer (see text). To minimize the jitter of the mode, the optics of the laser were carefully mounted on an NRC vibration-isolation table. 
in Figure 2.

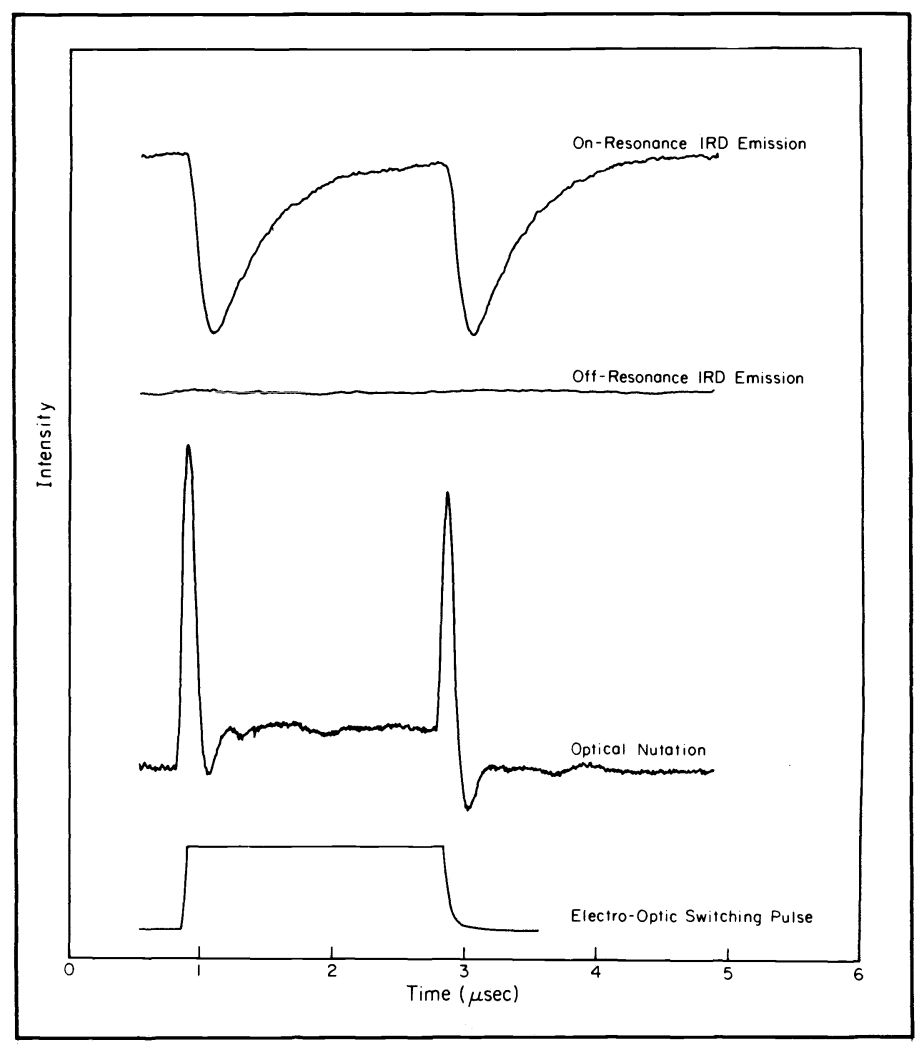

Figure 2. The boxcar output plots of the on-resonance and off-resonance emission decay when the electro-optic switching pulse is on and off. Also shown in this figure is the optical nutation of iodine at $0 \mathrm{C}$. The onresonance emission was taken at $14 \mathrm{MHz}$ switching frequency while the nutation was taken at $4 \mathrm{MHz}$.

\section{The Coherent Transients In Solids}

The experimental setup for the solid state experiments is identical to IRD except we monitored the laser intensity in the forward direction. Carefully mounted photodiodes were used in combination with filters to obtain the transients. These transients will appear either as coherent absorption and emission, as in the optical nutation (see Figure 2), or as beating with the incident beam, as in the free induction decay (Figure 3), sig-

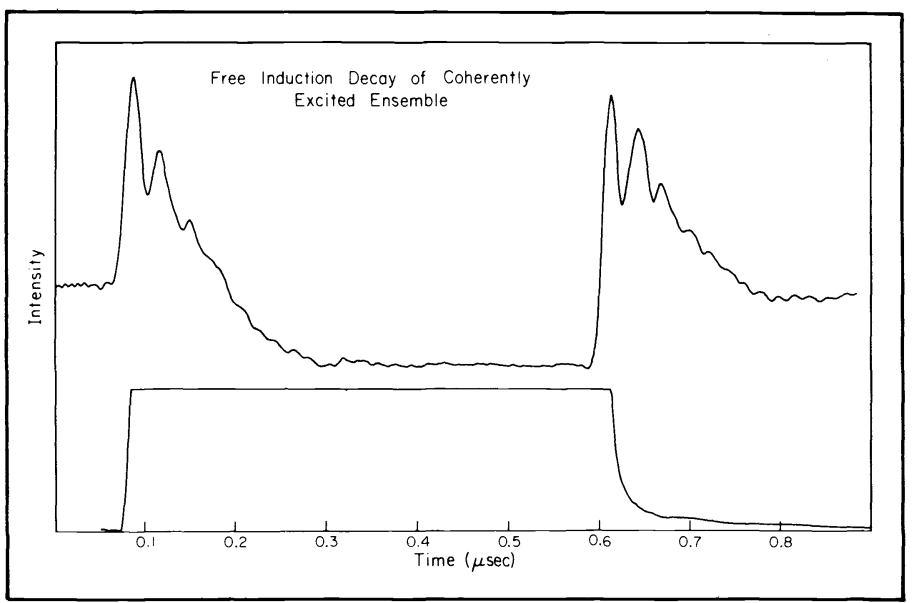

Figure 3. The free induction decay of iodine gas with the cold finger at O C (switching frequency is $38 \mathrm{MHz}$ ) coinciding with the leading and trailing edges of the EOC pulse. nal. ${ }^{16}$ The output of the biased diode (with constant voltage supply) was amplified and fed into either an oscilloscope or the boxcar integrator. The laser power was measured by a Spectra Physics power meter (Model 401B). Knowing the power of the single mode and the beam diameter gave the radiation density at the sample. While the coherent transients in the gas phase ${ }^{11}$ are very sensitive to the pressure in the cell, the coherent transients of the solid depend crucially on the crystal temperature, as evident from our work on pentacene in P-terphenyl between $1.7 \mathrm{~K}$ and above the $\lambda$-point of liquid helium.

\section{Optical $T_{1}$ and $T_{2}$ Processes of Excited States}

Using the above techniques in this series of experiments and in other experiments that will be published elsewhere, we are investigating in a systematic way the optical $T_{1}$ and $T_{2}$ processes. The small, intermediate, and large molecule limits, both at low pressures and in the solid phase, are examined. The objectives are to obtain information about the incoherent and coherent decays from this new technique and to correlate them with the findings of the many theories that already exist about the nature of primary excited states.

Population Distribution of the Inhomogeneous Gaseous Ensemble. We start with the prototype $\mathrm{I}_{2}$ molecule where we expect a clean excitation distribution and minimum vibronic scrambling. Naturally we must consider the Doppler broadening of the transition resonances $\left(\mathrm{T}_{2} *\right)$. Another important system, which certainly is not as simple electronically as iodine, is $\mathrm{NO}_{2}$ on which decades of work have already been spent to understand its spectroscopy.

The $\mathrm{I}_{2}$ gas was excited at $5897.5 \AA$ and Figure 2 shows the decay of the iodine on-resonance and off-resonance when the electro-optic switch is modulating the laser beam. The gas pressure was controlled by using different temperature baths at the cold finger. Typically, 30 mtorr was used. At this pressure both the coherent transients and IRD were monitored. We also checked the signal at liquid nitrogen temperature and at room temperature and no decay was observed.

Two sub-ensembles must be considered in treating the decay and dephasing of the dipoles in the inhomogeneous ensemble. The first group of molecules consists of those that were originally excited by the single mode $\mathrm{cw}$ laser. The degree of inversion after long times (compared with relaxation times) can be obtained from Eqs. (8)-(10). On-resonance and if the laser intensity is sufficiently large, $R_{3} \sim 0$. Turning the pulse on with $\Delta \gg 1 / \mathrm{T}_{2}$, this group of molecules will decay freely while a new group of molecules having $R_{3}^{0}=-1, R_{2}^{0}=R_{1}^{0}=0$ will be coherently driven. Hence, one expects the emission intensity, which monitors the diagonal elements of the density matrix, to build up and decay.

In the iodine case, the IRD fast decay constant was found to be $2.3 \mu \mathrm{sec}^{-1}$ at $100 \mathrm{mtorr}$. Stern and Volmer ${ }^{17}$ have shown that the total decay may be partitioned into a radiative part and another part which measures the number of deactivating collisions. The latter depends on the pressure of the gas and the molecular collision diameter $\sigma$. Using our results we obtain a radiative decay constant of $1.3 \mu \mathrm{sec}$ which is in excellent agreement with the results of phase fluorometry ${ }^{18}$ done on $I_{2}$ gas. By lowering the gas pressure (almost a collisionless gas), the decay time constant gets longer as expected from simple radiationless decay theory. Currently we are investigating the nature of the decay as a function of pressure and laser frequency. This will enable us to establish the relationship between the effective density of states ${ }^{19}$ and the excess rovibronic energy in intervals of $10^{-4}$ wavenumbers. Hence, the sparse intermediate limit ${ }^{20}$ (as opposed to the statistical or resonance limits) for the coupling between the prepared state and the rovibronic continua can be clearly identified. The beating between the different levels of the system during the decay can also be 
seen since our switching procedure is, perhaps, fast enough to give the appropriate time resolution. As mentioned before, the IRD experiments were also tried at $77 \mathrm{~K}$ and at room temperature (where a very strong emission was seen) and no signal was detected.

For $\mathrm{NO}_{2}$ the situation is different from iodine. The density of vibrational ground states that are quasiresonant with the excited level is higher. Moreover, these nonlinear molecules undergo relatively large geometrical changes when excited. This results in a large vibrational Franck-Condon overlap factor which makes the vibronic coupling matrix elements rather large and hence a short decay time is expected. At room temperature the Doppler width of $\mathrm{NO}_{2}$ near $4880 \AA$ is $\sim 1 \mathrm{GHz}$. In our experiments the laser excites $\mathrm{NO}_{2}$ at $5935.7 \AA$ where the absorption and fluorescence $(0.1$ mtorr) at low resolution are known. The coherent decay at relatively high pressure is very fast $(\lesssim 65$ nsec) and again disappears at low temperatures. We hope to report soon on the complete study of $\mathrm{NO}_{2}$ decay characteristics at different pressures and excitation energies.

Coherent Pumping and the Optical Transition Moment. As we described before, in the rotating frame and on-resonance the macroscopic moment [see Eqs. (8)-(11)] will nutate optically at Rabi's frequency. ${ }^{22}$ Hence, if we know the transition rate for the coherent pumping and the electric field strength at the sample, we can compute the transition moment quite easily:

$$
\left(\mu \cdot \mathrm{E}_{0} / \hbar\right)=\text { oscillation frequency }
$$

Knowing the laser intensity (after the first glass window of the cell) and measuring the width of the beam profile (FWHM) at the iodine cell gave $\mathrm{E}_{0}=105 \mathrm{~V} / \mathrm{cm}$. These preliminary results therefore give $\mu=0.05$ Debye for the resonance transition. To our knowledge, this is the first time that this quantity has been measured directly for an electronic transition. ${ }^{23}$

The coherent pumping rate on-resonance can also be used to obtain information about the dipole dephasing processes. From the results of iodine at relatively low pressure (gas temperature is near $0 \mathrm{C}$ ), we obtained a pumping rate of $2.1 \times 10^{6} \mathrm{c} / \mathrm{sec}$ for the molecular packets that are driven on resonance. This value gave a $\mathrm{T}_{2}$ that predicts a free induction decay time constant of $\approx 50 \mathrm{nsec}$ since

$$
\frac{1}{\tau}=\mathrm{T}_{2}^{-1}+\left[\mathrm{T}_{1} \mathrm{~T}_{2}^{-1}\left(\frac{\mu \cdot \mathrm{E}_{0}}{\hbar}\right)^{2}+\mathrm{T}_{2}^{-2}\right]^{1 / 2}
$$

This is indeed in good agreement with our results (see Figure 3), thus ensuring the self-consistency and showing that this simple technique can be used for examining optical phase coherence and incoherent decay processes in excited states.

The Phenomenon of Optical Coherence in Molecular Solids. Using the above techniques we have observed for the first time the coherent optical ringing of molecular crystals in their excited states. Several systems (ionic and molecular) are being investigated in our laboratory and we present here the data on pentacene in p-terphenyl at $1.7 \mathrm{~K}$.

Pentacene in p-terphenyl is known to show four distinct sites in the absorption and emission, with a linewidth of $1-3 \mathrm{~cm}^{-1}$ at $1.8 \mathrm{~K}^{24}$ These origins fluoresce when the laser is on-resonance. The so-called $\mathrm{O}_{1}$ origin was excited with a single mode of the dye laser and fluorescence was seen while monitoring the coherent transients of the $\mathrm{O}_{1}$ resonance. When the electro-optic crystal was triggered so the laser was switched out of a particular "homogeneous molecular packet," we observed the transients shown in Figure 4. These signals were absent when the laser beam was blocked before the crystal and also when the crystal was out of the optical path. The fast transient, together with the IRD signal, disappeared at a transition temperature of Approximately $3 \mathrm{~K}$. This is expected since the optical spectral diffusion rate will increase when the phonon population increases. Of course this is being studied now in much more detail.
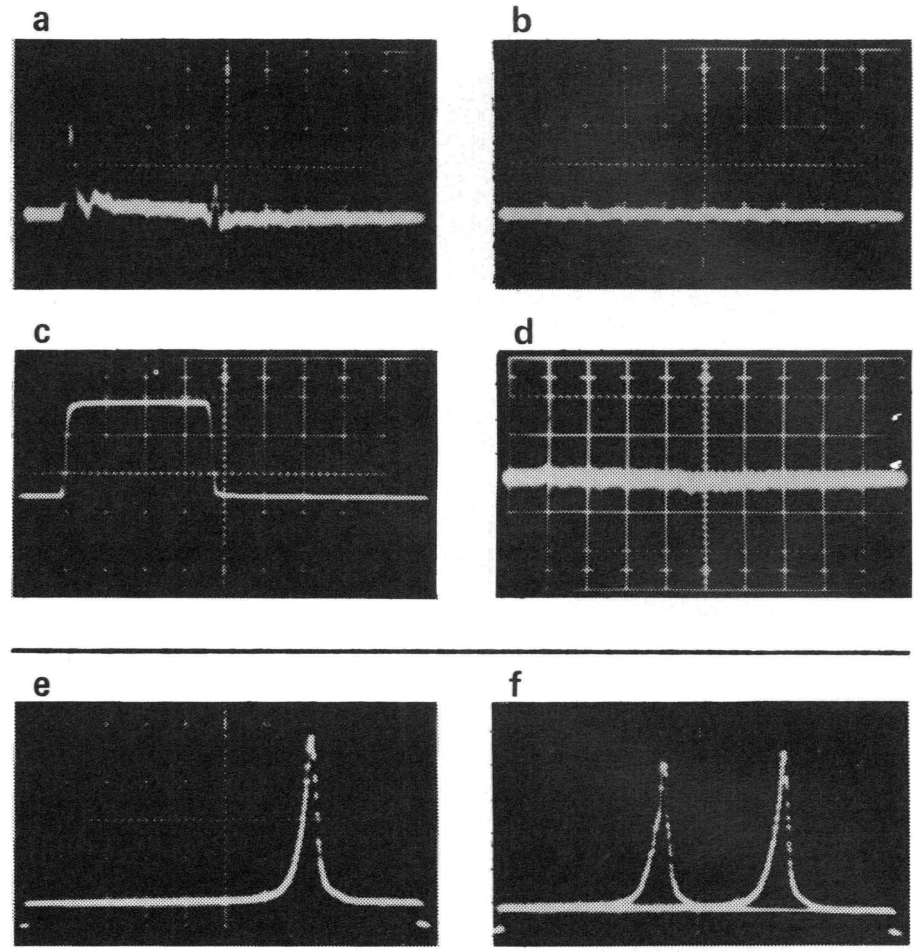

Figure 4. Coherent optical ringing from solid pentacene in p-terphenyl at $1.7 \mathrm{~K}$. The time resolution is $100 \mathrm{nsec} /$ division. (c) is the EOC pulse and (e) and ( $f$ ) are the mode structure when the pulse is off and on. (b) is the output when the laser beam is blocked and (d) is the output (at high sensitivity) without the crystal in place.

The emission signal detected at a right angle follows the principles discussed before for the incoherent resonance decay observed $^{25}$ in gases. In the solid the IRD pattern depends on the laser power and $\Delta$. From these preliminary results we obtain a homogeneous linewidth for the 0,0 origin of $13 \mathrm{MHz}$ which is orders of magnitude smaller than the apparent optical width and the homogeneous vibronic width. This implies that dephasing of molecular dipoles in the solid at $1.7 \mathrm{~K}$ is significant and competes with the spontaneous emission of the whole excited manifold. We should add that the forward coherent signal was not observed when the laser mode was on-resonance with the vibronic origin near $5829 \AA$. This observation ${ }^{26}$ is in agreement with the conclusion that a fast decay process does exist ${ }^{24 a}$ in the vibrationally excited states of the solid, thus implying the crucial dependence of vibrational relaxations on the excess vibronic energy in the mixed crystal.

\section{Optical Coherence of Molecular Beams ${ }^{27}$}

We have used the IRD method to detect transients of $I_{2}$ molecules in a collisionless molecular beam. The results indicate that the optical coherence is lost by the spontaneous decay of the system. The optical $\mathrm{T}_{1}$ in the beam was found to be $1.24 \pm$ $0.02 \mu \mathrm{sec}$ which is in excellent agreement with the Stern-Volmer zero-pressure time constant observed in the gas bulb. The optical free induction decay in the beam (average velocity of $1.6 \mathrm{x}$ $10^{4} \mathrm{~cm} / \mathrm{sec}$ ) was also observed. ${ }^{28}$ Quantum beats between the emitted light and the switched laser frequency were seen on the decay. This optical non-linear effect in beams has never been observed before. The beat frequency follows the electro-optical switching frequency while the decay is consistent with the conclusion that $\mathrm{T}_{1} \approx \mathrm{T}_{2}$ in the collisionless beam. 


\section{Acknowledgments}

I wish to thank Drs. J. Morris Weinberg and T. Hirschfeld for the invitation to present this work at the 20th Annual Symposium on Optical, Electro-optical, Laser and Photographic Technology (sponsored by the Society of Photo-Optical Instrumentation Engineers, SPIE) in August of 1976. I should point out that without the hard (late hours) work of T. Orlowski, $\mathrm{K}$. Jones, and D. Dawson of this laboratory, this work would never have been completed. This work was supported in part by the President's Venture Fund and in part by the Sloan Foundation.

\section{References}

1. W. Weisskopf and E. Wigner, Z. Physik, 63, 54 (1930).

2. A. Lorentzian transition that reflects the true spectral broadening is a homogeneous resonance.

3. For a review see: Excited States, Vol. 2, edited by E. Lim (Academic Press, New York, 1975)

4. W. E. Lamb, Jr., Phys. Rev., 55, 234(A) (1939).

5. These processes are similar to NMR relaxations; $T$ is the time constant for the decay of excited levels and $T_{2}$ is the time during which the excited state has a definite phase memory.

6. F. Bloch, Phys. Rev., 70, 406 (1946).

7. E. L. Hahn, Phys. Rev., 77, 297 (1950).

8. N. A. Kurnit, I. D. Abella, and S. R. Hartmann, Phys. Rev. Lett., 13 567 (1964).

9. N. Takeuchi and A. Szabo, Phys. Lett., 50A, 361 (1974); T. Aartsma and D. A. Wiersma, Phys. Rev. Lett., 36, 1360 (1976).

10. C. L. Tang and H. Statz, Appl. Phys. Lett., 10, 145 (1968); G. B. Hocker and C. L. Tang, Phys. Rev. Lett., 21, 591 (1969).

11. R. G. Brewer and R. L. Shoemaker, Phys. Rev. A, 6, 2001 (1972); R. Brewer and A. Genack, Phys. Rev. Lett., 36, 959 (1976).

12. S. L. McCall and E. L. Hahn, Phys. Rev. Lett., 18, 908 (1967).

13. R. H. Dicke, Phys. Rev., 93, 99 (1954).
14. R. P. Feynman, F. L. Vernon, Jr., and R. W. Hellworth, J. Appl. Phys., 28, 49 (1957).

15. J. Telle and C. L. Tang, Appl. Phys. Lett., 24, 85 (1974); ibid., 26 572 (1975).

16. R. Brewer and A. Genack (Ref. 11) have reported recently the forward coherent transients in iodine. Our results on the coherent decay are in agreement with theirs.

17. O. Stern and M. Volmer, Z. Physik., 20, 183 (1919)

18. L. Brewer, R. Berg, and G. Rosenblatt, J. Chem. Phys., 38, 1381 (1963); A. Chutjian, Ph.D. Thesis, University of California, Berkeley (1966).

19. The effective density of states here means the product of the actual density of states times the off-diagonal matrix element that connects the primary Born-Oppenheimer state with the continuum.

20. (a) G. W. Robinson and R. P. Frosch, J. Chem. Phys., 38, 1187 (1973); M. Bixon and J. Jortner, ibid., 48, 715 (1968); D. Chock, J. Jortner, and S. A. Rice, ibid., 49, 610 (1968); (b) For excellent reviews by S. A. Rice and G. W. Robinson on the subject, see Excited States, edited by E. C. Lim (Academic Press, New York, 1974 and 1975), Vols. I and II.

21. C. G. Stevens, M. W. Swagel, R. Wallace, and R. N. Zare, Chem. Phys. Lett., 18, 465 (1973).

22. R. L. Shoemaker and E. W. van Stryland, J. Chem. Phys., 64, 1733 (1976).

23. We are now making a complete fit for the coherent decay curve so an accurate measurement of the dipole moment can be obtained.

24. (a) A. P. Marchetti, W. C. McColgin, and J. H. Eberly, Phys. Rev. Lett., 35, 387 (1975); (b) J. H. Meyling and D. A. Wiersma, Chem. Phys. Lett., 20, 383 (1973).

25. A. H. Zewail, T. E. Orlowski, and D. R. Dawson, Chem. Phys. Lett., 44, 379 (1976).

26. When the laser was near the $5829 \AA$ transition, the crystal was fluorescing, but not as strongly as when the laser was near the $O_{1}$ origin at $5921.7 \AA$.

27. A. H. Zewail, T. E. Orlowski, R. Shah, and K. E. Jones, Chem. Phys. Lett., to be published. 28. A. H. Zewail, T. E. Orlowski, R. R. Shah and K. E. Jones (to be
published). 